\title{
Discrimination of what, when, and where is not based on time of day
}

\author{
STEPHANIE J. BABB and JONATHON D. CRYSTAL \\ University of Georgia, Athens, Georgia
}

\begin{abstract}
Rats ( $n=6$ ) visited four baited locations (randomly chosen on each trial; study phase), one of which was randomly selected to provide chocolate. After short (1-h) or long (25-h) retention intervals (RIs), eight locations were available, and the four locations not available in the study phase provided food (test phase); the chocolate location also provided food after long RIs. More visits to the chocolate location occurred after long RIs than after short RIs. Next, chocolate was paired with LiCl during the long RI (i.e., after encoding the chocolate location). Fewer revisits to the chocolate location occurred after $\mathrm{LiCl}$ than in previous testing with the long RI. The rats demonstrated complete transfer when grape replaced chocolate after $\mathrm{LiCl}-$ chocolate pairing. The discrimination of what, when, and where could not be based on adopting different revisit strategies at different times of testing.
\end{abstract}

Tulving (1972) proposed a distinction between episodic memory and semantic memory. Episodic memory refers to the encoding and retrieval of personal, unique past experiences. In contrast, semantic memory is knowledge of general facts, such as knowing that the earth is round. Tulving's (1972) classic definition of episodic memory states that "Episodic memory receives and stores information about temporally dated episodes or events, and temporal-spatial relations among these events" (p. 385). According to this definition, episodic recall involves retrieval of information about three aspects of an event or episode: what occurred, when it transpired, and where it took place (what-when-where, or WWW).

The definition of episodic memory has been refined to include experiences such as an awareness that the event happened in the past, or chronesthesia; a feeling of reexperiencing the past event, or autonoesis; and a realization that the memories belong to oneself, or knowledge of self (e.g., Tulving, 1983, 2005; Tulving \& Markowitsch, 1998). Unfortunately, these terms are difficult, if not impossible, to test in nonverbal animals (Griffiths, Dickinson, \& Clayton, 1999; W. A. Roberts, 2002; Suddendorf \& Busby, 2003; Suddendorf \& Corballis, 1997). Because focusing on WWW captures only a subset of the current definition of episodic memory, the study of WWW in nonhuman animals is referred to as "episodic-like" (Clayton \& Dickinson, 1998).

This work was supported by National Institute of Mental Health Grants MH64799 and MH64799-S1. This research was completed in partial fulfillment of a doctoral degree to S.J.B. at the University of Georgia. We thank Rob Hampton for advice about the study and Kenneth W. Maxwell for technical assistance. The raw data (time of occurrence of each stimulus and response) are available at www.uga.edu/animal-cognition-lab. S. J. Babb is now at the Department of Neurobiology and Anatomy, University of Texas Health Science Center Houston. Correspondence should be addressed to J. D. Crystal, Department of Psychology, University of Georgia, Athens, GA 30602-3013 (e-mail: jcrystal@uga.edu).
Clayton and colleagues (Clayton, Bussey, \& Dickinson, 2003) have argued that tests of episodic memory in animals should focus on the behavioral aspects of episodic memory, instead of the phenomenological experiences that may accompany these memories. They proposed three criteria: content, structure, and flexibility. According to Clayton and colleagues, knowledge of what, when, and where is evidence for episodic-like memory if the structure is an integrated representation that can be used flexibly (Clayton, Bussey, \& Dickinson, 2003; Clayton, Bussey, Emery, \& Dickinson, 2003; de Kort, Dickinson, \& Clayton, 2005). Clayton, Bussey, and Dickinson argued that an integrated representation of what, when, and where means that retrieval of any one feature of an episode automatically retrieves the other features. This integrated structure permits the discrimination of multiple events that share some, but not all, features. The memory must also be flexibly updated and generalized over situations.

Some birds cache food and recover it days, weeks, or months later. The recovery of stored food is guided by memory for the spatial location of the cache site and also memory for the content of the caches, and these memories are encoded during a single brief caching episode (e.g., Clayton \& Dickinson, 1998; Shettleworth, 1998). Clayton and Dickinson (1998) provided the first evidence of discrimination of WWW in food-storing scrub jays. They provided jays with the opportunity to cache peanuts or wax worms. The birds either stored peanuts followed by worms or, on other trials, stored worms followed by peanuts. The opportunity to retrieve the cached foods occurred after either a short or a long RI. For some birds, the worms were decayed after the long RI, and for other birds, they were replenished with fresh worms (peanuts did not decay). The birds preferred the worm rather than peanut cache sites when the worms were fresh, but they reversed this preference when the worms were decayed. These data suggest that the jays were sensitive to what (food type), when (time of caching and recovery), and where (location 
in the tray). Since this initial demonstration, Clayton and colleagues have established a growing body of research to indicate that scrub jays have a detailed representation of what, when, and where food was cached. For example, jays (1) remember the specific food types that they cache and recover (Clayton \& Dickinson, 1999a, 1999b), (2) remember the relative time and location of caches (Clayton \& Dickinson, 1999b), (3) form integrated memories for the location and time of caching of particular foods (Clayton, Yu, \& Dickinson, 2001), and (4) flexibly update information about caching episodes with new information acquired during the RI (Clayton, Yu, \& Dickinson, 2003). Changing the expected value of the to-be-recovered food item by degrading it (i.e., decreasing its value; Clayton \& Dickinson, 1998, 1999b; Clayton, Yu, \& Dickinson, 2001, 2003), ripening it (i.e., increasing its value; de Kort et al., 2005), and satiation of that food type (i.e., decrease in value; Clayton \& Dickinson, 1999a, 1999b) have been used to demonstrate discrimination of WWW. Clayton and colleagues have argued that the relative familiarity of the tray cues from the caching episode cannot explain the discrimination of WWW.

We recently used Clayton's behavioral approach to test rats' ability to discriminate WWW in an eight-arm radial maze (Babb \& Crystal, 2005). Daily testing was divided into forced choice and free choice phases, which were separated by either a short $(0.5-\mathrm{h})$ or a long (4-h) RI. In the forced choice phase, the rats were required to visit four baited locations (randomly chosen on each trial for each rat), one of which was randomly selected to provide chocolate. The rats were trained to discriminate between short and long RIs. After the short RI, the four locations not seen in the forced choice phase provided regular food. On days with a long RI, the free choice phase was identical to the short RI condition, except that the chocolate arm was replenished (i.e., provided an additional chocolate pellet). On any given day, either the short or the long RI (but not both) was tested. We found that rats revisited the chocolate location more after the long RI than after the short RI. Chocolate was then paired with $\mathrm{LiCl}$ in an attempt to reduce the likelihood of a revisit to the chocolate location after the long RI; the rats were given three separate pairings of chocolate and $\mathrm{LiCl}$ outside of regular testing (i.e., in the intertrial interval [ITI]), which eliminated the preference for chocolate. The animals were subsequently tested using the long RI (i.e., a condition in which the rats had previously revisited the chocolate location at a high rate). The rats made fewer revisits to the chocolate location after the $\mathrm{LiCl}$ treatment than in previous testing with the long RI. The animals could not have reduced the rate of revisits to the chocolate location without discriminating WWW. Because chocolate revisits declined after $\mathrm{LiCl}$ treatment, in conditions that controlled the level of relative familiarity of chocolate, discrimination of WWW is a single parsimonious interpretation for the selective revisits to chocolate before $\mathrm{LiCl}$ treatment and the decline in visits to chocolate after $\mathrm{LiCl}$ treatment.

Hampton, Hampstead, and Murray (2005) argued that our rats could have solved the discrimination of WWW by using time of day. Our rats were always tested in the forced choice phase in the morning, and the free choice phase occurred in the morning or afternoon after the short or long RI, respectively. Therefore, the rats may have used a representation of time of day from a circadian oscillator (e.g., Mistlberger, 1994) to discriminate between the short and long RIs. Consequently, the rats could have adopted different revisiting strategies in morning and afternoon tests. Hampton et al. argued that this strategy was not sufficient to demonstrate "when" memory in the sense that is required for WWW memory. In contrast, Hampton et al. argued that a demonstration of "when" memory involves requiring the animal "to know how long ago they learned the locations of food, rather than what time of day is associated with the availability of a certain food" (p. 256), as has been demonstrated by Clayton and colleagues. Moreover, Hampton et al. pointed out that encoding a rule about the availability of chocolate at different times of day is analogous to semantic, rather than episodic, memory.

Hampton et al. (2005) also emphasized the importance of documenting that the discrimination of WWW is acquired through experience with the contingencies of the experiment. The influence of experience may be evaluated by comparing the preference for revisits at the beginning and end of training. Presumably, there would not be a preference for revisits to the chocolate location in the initial block of training. However, by the end of training, we would expect to document a reliable preference; these two predictions may also be evaluated by testing the interaction of training and RI.

\section{EXPERIMENT 1}

In the present study, we controlled for time of day by using RIs of $1 \mathrm{~h}$ (short) and $25 \mathrm{~h}$ (long). For example, a rat that was tested in the forced choice phase beginning at approximately $1200 \mathrm{~h}$ would be returned to the maze for the free choice phase at approximately $1300 \mathrm{~h}$ the same day (short RI) or $1300 \mathrm{~h}$ the next day (long RI). Therefore, the rats always received the free choice phase at the same time of day. In a separate experiment, we examined forgetting functions of spatial memory and found that rats' accuracy in the radial maze was still high $(62 \%)$ after $25 \mathrm{~h}$ (Crystal \& Babb, 2006). The ITI was at least 48 h, in order to reduce similarity of the RI and ITI. After the animals had learned to selectively revisit the chocolate location, chocolate was paired with $\mathrm{LiCl}$. However, in the present study, the taste aversion manipulation was inserted into the RI; the rats were given a forced choice phase, and during the 25-h RI, they were fed chocolate and injected with $\mathrm{LiCl}$ before completing the free choice phase. Because the animals encountered chocolate prior to $\mathrm{LiCl}$ treatment, avoiding chocolate after $\mathrm{LiCl}$ treatment could not be explained by failure to encode the location of chocolate. A reduction in visits to the chocolate location after encoding would document some degree of flexibility to update memory on the basis of newly acquired information (i.e., that chocolate is bad). 


\section{Method}

Subjects. Six male Long Evans rats (Harlan, Madison, WI) were individually housed in a colony with light onset at $0700 \mathrm{~h}$ and offset at $1900 \mathrm{~h}$. The rats were given unlimited access to 5001 Rodent Diet (Lab Diet, Brentwood, MO) for 1 week, and then given $20 \mathrm{~g}$ for 1 day and $15 \mathrm{~g}$ per day on subsequent days. Water was available at all times, except during brief testing periods. The rats were approximately 77 days old at the beginning of the experiment; their average weight was $239 \mathrm{~g}$.

Materials. Testing was conducted in an eight-arm radial maze. The central hub (white polypropylene octagonal base [28.6- $\mathrm{cm}$ diameter, $11.4-\mathrm{cm}$ sides], metal walls [33.3 $\mathrm{cm}$ high], and a clear polycarbonate lid, Med Associates, ENV-538) was equipped with eight computer-controlled guillotine doors (ENV-540). The arms (76.2 $\mathrm{cm}$ long and $8.9 \mathrm{~cm}$ wide, with $17.5-\mathrm{cm}$ high clear polycarbonate walls and topped with polycarbonate) radiated from the hub with equal spacing between arms. A food trough (ENV-200R1M) was placed at the end of each arm. A photobeam (ENV-254, approximately $1 \mathrm{~cm}$ inside each food trough, $1 \mathrm{~cm}$ from the trough's bottom) detected head entries. A 45-mg pellet dispenser (ENV-203) was placed behind each food trough. Additional photobeams were located in each arm 3.8 and $5.1 \mathrm{~cm}$ from the guillotine doors.

The maze was positioned on stools $81.3 \mathrm{~cm}$ above the floor. White noise $(67 \mathrm{~dB}$ from a speaker located in the ceiling above the hub) minimized outside noise. A 500-MHz computer in an adjacent room, running MED-PC for Windows (Version 4.1), controlled experimental events (guillotine doors and food) and recorded the data (photobeam breaks) with $10-\mathrm{msec}$ resolution. A video camera in the ceiling above the center of the maze was used to observe the rats.

Procedure. Pretraining consisted of 3 days in which three pellets (PJA/I-0045, Research Diets Inc., New Brunswick, NJ) were placed along each arm and one pellet was placed in each food trough to provide the animals with the experience of obtaining food in the maze. Each day, one randomly chosen arm contained chocolate pellets (F0299, Bio-Serv, Frenchtown, NJ). The rats were individually placed on the maze for an average of approximately 10.0, 10.0, and 7.7 min on Days 1, 2, and 3, respectively.

During initial training, the rats were individually placed in the central hub beginning at $1200 \mathrm{~h}$; all eight doors were then opened. The session ended when the animals had depleted all arms or $10 \mathrm{~min}$ had elapsed. Training was conducted once per day for 10 trials. Trials were separated by at least a 48-h ITI.

For the remainder of the study, testing consisted of two shifts per day for each rat. The rats were individually placed in the maze beginning at $1200 \mathrm{~h}$ for Phase 1 (forced choice). Four doors (randomly chosen for each rat each day) were then opened, with the restriction that one of the arms dispensed chocolate pellets (chocolate arm); all other arms dispensed regular pellets. A pellet was delivered to each of the accessible food troughs contingent on the first head entry recorded by the photobeam located in each of the troughs. The animals were later returned for Phase 2 (free choice) in which all eight doors were open. The interval between the forced and the free choice phases served as a discriminative cue. On days with a short RI, the interval between Phases 1 and 2 was $1 \mathrm{~h}$, and the only arms that provided food were the four arms not available in Phase 1; regular pellets were dispensed at these Phase 2 locations. On days with a long RI, the interval between Phases 1 and 2 was $25 \mathrm{~h}$; the free choice phase after a long RI was identical to the short RI condition, except that the location that had provided chocolate in Phase 1 also provided a chocolate pellet in Phase 2 (i.e., the chocolate arm was replenished). The rats were required to visit each arm to collect the remaining pieces of food after an RI; the free choice phase ended after the four or five pellets were collected in the short or the long RI, respectively. On any given day, either the short or the long RI (but not both) was tested. Each trial was separated by at least a 48-h ITI.

The rats received alternating blocks of short RI followed by blocks of long RI sessions. Each block differed only in RI and consisted of
$10-15$ days of training. A total of 44 short RI and 43 long RI training sessions were conducted. The animals were then given 16 days of mixed short and long RI conditions. The order of short and long RI was randomized across sessions in the mixed condition.

Next, the rats received pairings of chocolate and $\mathrm{LiCl}$. Ten minutes after all rats completed a Phase 1 shift (i.e., during the RI), the animals were given access to $50 \mathrm{~g}$ of chocolate pellets for $30 \mathrm{~min}$, and $10 \mathrm{~min}$ after removal of the food, they were injected with an isotonic solution of $\mathrm{LiCl}$ in distilled water $(0.75 \mathrm{~mol} / \mathrm{L}, 0.6 \mathrm{ml} / 100 \mathrm{~g}$ of body weight i.p.). The animals were returned to the maze $25 \mathrm{~h}$ after Phase 1 for a long RI Phase 2 shift; the Phase 2 shift was identical to previous free choices after long RI, except that the shift ended after the fourth regular pellet was dispensed or $20 \mathrm{~min}$ had elapsed. Two trials with $\mathrm{LiCl}$ treatment were conducted.

Throughout the experiment, the arms of the maze were cleaned with Nolvasan (Fort Dodge Animal Health, Fort Dodge, IA) each day between forced and free choice phases. A plastic bag with holes and filled with chocolate and regular pellets was taped on the stool at the end of each arm beside the filled pellet dispensers (i.e., food odors were constant throughout all parts of the experiment).

Throughout this article, the criterion for statistical significance is .05 .

\section{Results and Discussion}

During block testing with the short RI, the proportion of visits to the chocolate location in the first four choices was .22. During block testing with the long RI, the proportion of visits to the chocolate location in the first four choices was .51. The proportion correct after the RI is shown in Table 1; these data are based on an analysis that was restricted to the first four choices among the seven nonchocolate arms.

Figure 1 (top panel) plots the proportion of first-four choices that included chocolate during mixed short RI and long RI testing. The rats visited the chocolate site more often in the long than in the short RI conditions $[t(5)=$ 5.37, $p<.01$ ]. Because the data shown in Figure 1 (top panel) and Table 1 were derived from independent segments of the data (i.e., chocolate and nonchocolate arm entries), we used these data to compare error rates in the mixed condition. The probability of a revisit to a nonchocolate arm after a short RI (i.e., a baseline error rate) was $.05 \pm .02(M \pm S E M)$. Although this baseline was lower

Table 1

Mean Proportion Correct in the First Four Choices Excluding the Chocolate Location After the Retention Interval in Experiment 1

\begin{tabular}{|c|c|c|c|c|}
\hline \multirow[b]{3}{*}{ Trial Type } & \multicolumn{4}{|c|}{ Retention Interval } \\
\hline & \multicolumn{2}{|c|}{ Short } & \multicolumn{2}{|c|}{ Long } \\
\hline & $M$ & $S E M$ & $M$ & $S E M$ \\
\hline \multicolumn{5}{|c|}{ Experiment 1} \\
\hline Block & .87 & .02 & .68 & .01 \\
\hline Mixed & .95 & .02 & .65 & .03 \\
\hline Lithium chloride & & & .75 & .06 \\
\hline \multicolumn{5}{|c|}{ Experiment 2} \\
\hline Initial & .90 & .06 & .75 & .08 \\
\hline Terminal & .94 & .04 & .70 & .03 \\
\hline
\end{tabular}

Note-The analysis of the first four choices was restricted to the seven nonchocolate arms. 

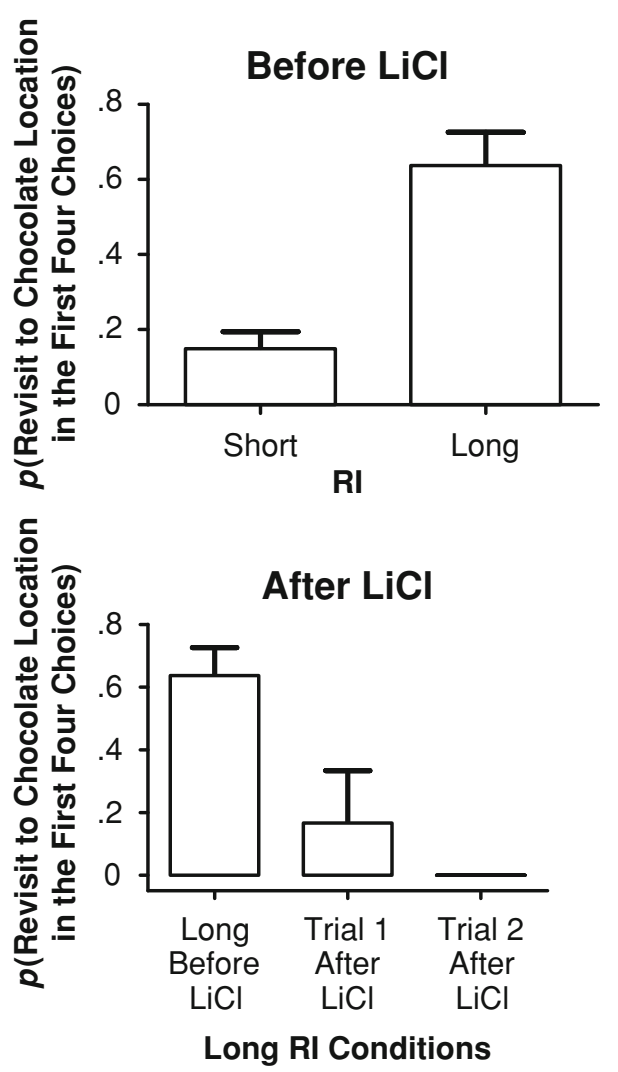

Figure 1. Mean proportion of chocolate revisits in the first four choices of Phase $\mathbf{2}$ in mixed testing is plotted as a function of retention intervals (RIs) in Experiment 1. 'Top and bottom panels display data from testing without and with $\mathrm{LiCl}$ treatment, respectively (see text for details). Error bars represent SEM.

than the probability of a revisit to the chocolate location after a short RI, this difference was not statistically significant $[t(5)=2.24, p>.05]$. Therefore, after short RIs, the rats revisited the chocolate locations at a level comparable with the revisit rate for nonchocolate locations. In contrast, after long RIs, the rats revisited the chocolate location at a higher level than would be expected on the basis of revisits to nonchocolate arms. In particular, the probability of a revisit to a nonchocolate arm after a long RI (i.e., baseline error rate) was $.35 \pm .03$. The probability of a revisit to the chocolate location after a long RI was significantly higher than this baseline error rate $[t(5)=$ $3.01, p<.05]$. In summary, revisits to the chocolate location occurred a rate higher than expected from the baseline error rate after long, but not short, RIs.

Performance from the long RI in testing after the $\mathrm{LiCl}$ treatment is shown in Figure 1 (bottom panel) together with the expected probability of revisiting chocolate (i.e., long $\mathrm{RI}$ before $\mathrm{LiCl}$ treatment). The rate of revisits varied significantly across long RI conditions $[F(2,10)=6.74$, $p<.05]$. The revisit rate was significantly lower on the first $[F(1,10)=6.84, p<.05]$ and second $[F(1,10)=$ $12.55, p<.01]$ trials after $\mathrm{LiCl}$ relative to pre- $\mathrm{LiCl}$ test- ing. There was no statistical difference between the short RI before $\mathrm{LiCl}$ treatment (Figure 1, top panel) and the long RI after $\mathrm{LiCl}$ treatment (Figure 1, bottom panel) $[t(5)<1]$.

Because the post- $\mathrm{LiCl}$ testing in Phase 2 terminated after the fourth regular pellet had been dispensed, each rat did not encounter chocolate. After $\mathrm{LiCl}$ treatment, chocolate could be encountered on three occasions: first trial Phase 2, second trial Phase 1, and second trial Phase 2. In the first trial Phase 2, 4 of the rats encountered chocolate, and 3 of them ate it. In the second trial Phase 1, 6 rats encountered chocolate, and all 6 rats ate it. In the second trial Phase 2, 2 rats encountered chocolate, and 0 ate it. Therefore, encountering a chocolate pellet was not sufficient for a rat to reject eating it. Nevertheless, when tested after the RI, the rats avoided the location known to contain chocolate, reversing their initial preference to choose the chocolate location after a long RI.

Figure 2 documents that the discrimination of WWW was acquired through experience. The initial and terminal blocks consist of the first and last five trials, respectively, from short and long RI block training. There was a significant interaction between training and $\mathrm{RI}[F(1,5)=6.92$, $p<.05]$, reflecting a difference between the short and the long RI in terminal $[t(5)=4.00, p<.05]$ but not initial $[t(5)<1]$ training.

A preference test was given immediately after the final $\mathrm{LiCl}$ trial to evaluate the effectiveness of the taste aversion manipulation, using two bowls, each containing 50 chocolate or 50 regular pellets. The percentage of chocolate pellets consumed was $0.4 \% \pm 0.4$; each rat ate $100 \%$ of the regular pellets. By contrast, Babb and Crystal (2005) reported that rats not treated with $\mathrm{LiCl}$ consumed $97 \%$ of chocolate pellets when given the same two-bowl test.

In summary, the rats made more visits to the chocolate location after the long than after the short RI. Next, chocolate was paired with $\mathrm{LiCl}$ during the long RI. The rats made fewer revisits to the chocolate location after the $\mathrm{LiCl}$ treatment than in previous testing. The animals could not have reduced the rate of revisits to the chocolate location without knowledge of what, when, and where.

\section{EXPERIMENT 2}

In Experiment 2, we investigated whether the rats would exhibit transfer to a new distinctive food type (grape pellets) after chocolate was paired with $\mathrm{LiCl}$. The purpose of the transfer test was to evaluate the hypothesis that what the rats learned in Experiment 1 was specific to the chocolate food. Alternatively, the rats could have learned that the location with a distinctive food type was replenished. If the rats had learned that the distinctive food type was replenished, then we would expect that the revisit strategy would transfer to a new distinctive food type (grape pellets) without additional training. In contrast, if the revisit strategy was limited to the stimulus with which the rats had experience, then we would expect that the revisit strategy would not transfer to a new distinctive flavor. 


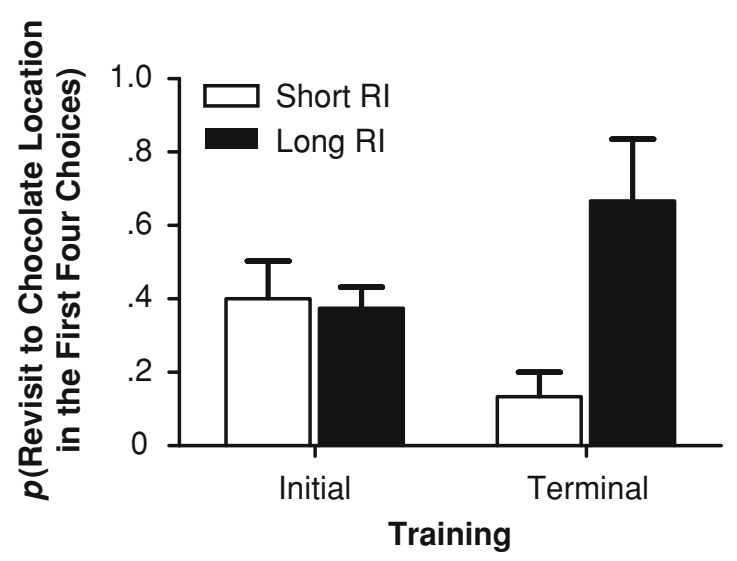

Figure 2. The discrimination of what-when-where was acquired through experience. The initial and terminal blocks consist of the first and last five trials, respectively, from short and long retention interval (RI) block training in Experiment 1. Error bars represent $S E M$.

\section{Method}

Subjects. The subjects were the rats used in Experiment 1, except that 1 animal was not tested because of illness.

Materials. The apparatus was the same as described in Experiment 1 .

Procedure. After completion of post-LiCl trials with chocolate, the rats received 22 days without testing, and the rats then received 12 trials of mixed short and long RI testing. The procedure was the same as that for the mixed condition in Experiment 1, except that grape was used as the distinctive pellet (i.e., grape replaced chocolate). The first and second trials of Experiment 2 were long and short RI trials, respectively.

\section{Results and Discussion}

The proportion correct after the RI is shown in Table 1 . Figure 3 shows that the rats exhibited complete transfer to grape distinctive pellets after the pairing of chocolate and $\mathrm{LiCl}$. In the first two trials (long and short $\mathrm{RI}$, respectively), revisits to the grape location were more frequent after the long than after the short RI $[t(4)=4.00, p<$ $.05]$ (Figure 3, top panel). During the remaining 10 trials, revisits to the grape location were more frequent after the long than after the short RI $[t(4)=9.80, p<.01]$ (Figure 3 , bottom panel). The magnitude of the difference in performance between the short and the long RIs in Experiment 1 (Figure 1, top panel) was not significantly different from the magnitude of the difference between performance in the short and long RIs in Experiment $2[t(4)=$ $2.41 ; t(4)<1 ; p \mathrm{~s}>.05$ ] (Figure 3 , top and bottom panels). The revisit strategy during training transferred to a new distinctive food, suggesting that what the rats learned in Experiment 1 was not specific to chocolate.

Because the probability of a revisit to the grape location after a long RI was higher in the initial test than it was for terminal performance $[t(4)=-9.37, p<.01]$, this apparent decline may reflect the growth of generalized flavor aversion over time; initial and terminal revisit probabilities after short RIs in Figure 3 did not differ $[t(4)=-0.65$, $p>$.05]. However, it is noteworthy that the decline in the probability of a revisit from initial to terminal tests after long RIs is primarily due to a higher than expected probability on the initial long RI. In particular, the probability of a revisit to grape in the initial long RI (Figure 3, top panel) was higher than the probability of a revisit to chocolate in Experiment 1 (Figure 1, top panel, long RI) $[t(4)=3.25$, $p<.05$ ]; note that this higher than expected probability is based on a single trial in which each rat revisited the grape location in the first four choices. By contrast, the probability of a revisit to grape in the terminal long RIs (Figure 3, bottom panel) was not reliably different from the probability of a revisit to chocolate in Experiment 1 (Figure 1, top panel, long RI) $[t(4)=-1.61, p>.05]$. In summary, the probability of revisiting the grape location after long RIs in Experiment 2 was at least as high as the probability of revisiting the chocolate location after long RIs in Experiment 1 (i.e., complete transfer was observed in both initial and terminal trials).

\section{GENERAL DISCUSSION}

In Experiment 1, rats were required to visit four randomly chosen baited locations, one of which was randomly selected to provide chocolate, during the study phase. The animals were later returned to the maze after either a short or a long RI, with all eight locations available. After the short RI, the four locations not available in the study phase provided food; after the long RI, the four locations not available in the study phase, plus the chocolate location,
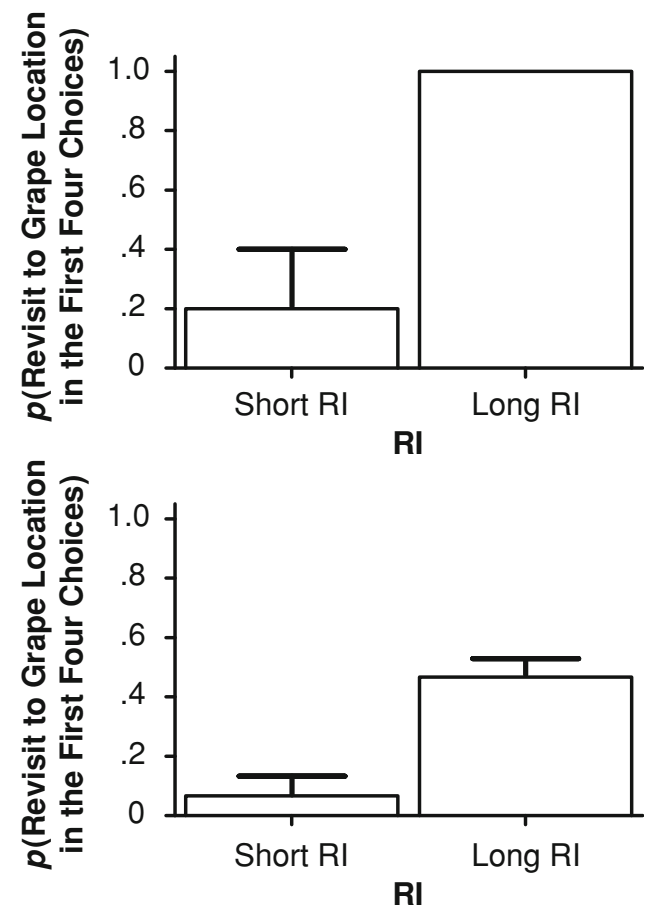

Figure 3. Mean proportion of revisits to the unique location (grape) during Phase 2 of the transfer test in Experiment 2. Top and bottom panels display data from the initial and terminal trials, respectively. Error bars represent $S E M$. RI, retention interval. 
provided food. The rats made more visits to the chocolate location after the long than after the short RI. Next, the animals received a taste aversion treatment during the long RI, in which chocolate was paired with $\mathrm{LiCl}$. The animals were subsequently tested at the end of the long RI (i.e., a condition in which the rats previously revisited the chocolate at a high rate). The rats made fewer revisits to the chocolate location after the $\mathrm{LiCl}$ treatment than in previous testing. The animals could not have reduced the rate of revisits to the chocolate location without knowledge of WWW.

In Experiment 2, grape replaced chocolate pellets after chocolate was paired with $\mathrm{LiCl}$. The rats exhibited complete transfer to grape distinctive pellets in both the first grape trials and in the subsequent mixed training with short and long RIs. The transfer data suggest that the revisit strategy learned in Experiment 1 was not specific to the chocolate food. Instead, the rats learned that the location with a distinctive food type replenished.

These results are consistent with the findings from our previous study (Babb \& Crystal, 2005). However, in that study, there was the possibility that the rats solved the discrimination of the WWW problem by using time of day. The rats in our earlier experiment were tested in the free choice phase in either the morning or the afternoon; therefore, they may have used a circadian representation of time of day to discriminate between the short and long RIs. Because in the present study we used 1- and 25-h RIs (i.e., test phases were conducted at the same time of day), time of day could not have served as a discriminative cue. Consequently, the discrimination of WWW in the present study satisfies Hampton et al.'s (2005) definition of "when" memory.

In Babb and Crystal (2005), chocolate was paired with $\mathrm{LiCl}$ during the ITI. By contrast, in the present study, chocolate was paired with $\mathrm{LiCl}$ during the long RI. Consequently, the observed reduction in revisits to the chocolate location (Figure 1) cannot be explained by a failure to encode the chocolate location after the chocolate was devalued. The revisiting strategy was acquired through experience with the replenishment contingency (Figure 2). Therefore, if the rats were responding to the RI as in prior training, they would have continued to revisit the chocolate location at a high rate. Consequently, the decline in revisits to chocolate documents some degree of flexibility to update memory on the basis of new information (i.e., chocolate is bad) acquired during the RI.

Roberts (2002) offered a rule-based criticism of Clayton and colleagues' demonstration of episodic-like memory that can be construed in order to describe our training data. According to Roberts's criticism, the memory trace for a chocolate location would be relatively strong after a short RI and relatively weak after a long RI. Therefore, the rat could adopt the rule "if the memory of the chocolate location is weak, revisit that location." However, the use of this rule does not predict the data observed after $\mathrm{LiCl}$ treatment. Testing after the $\mathrm{LiCl}$ treatment used a long RI. Therefore, application of the rule outlined above would predict revisits at a high rate, rather than the observed low rate. Nevertheless, if the rats learned the expectancy "if the memory of chocolate is weak, expect more chocolate," then the rats would not revisit the chocolate location because chocolate is repellent after $\mathrm{LiCl}$. Zentall (2005; Zentall, Clement, Bhatt, \& Allen, 2001) has also emphasized the need to document that episodic-like memory cannot be solved using rule-based learning.

Because there is no information about the subjective state of the animal when the animal makes a decision to revisit the chocolate location, it is possible that a threeway conditional discrimination of WWW occurs in the absence of any accompanying subjective states. One interpretation of the discrimination of WWW is that the rats retrospectively reexperience an event from memory to assess when it occurred in the past. However, the discrimination of WWW could also be based on an interval timing mechanism. According to the interval-timing hypothesis, discrimination of WWW is based on the discrimination of elapsing intervals with respect to a resetting stimulus (i.e., a pacemaker-accumulator mechanism; Church, Meck, \& Gibbon, 1994; Crystal, 2002). For example, short and long RIs can be discriminated by resetting an interval timing mechanism when the rats are handled at the start of each trial. To establish evidence that an animal represents "when" (rather than how long ago) an event has occurred, it will be necessary to rule out both the interval-timing and the circadian mechanisms. These hypotheses need further testing so that potential timing mechanisms in the discrimination of WWW can be evaluated.

The observation that rats discriminate WWW contrasts with other recent reports. W. A. Roberts and S. Roberts (2002) assessed the ability of rats to remember the order in which they entered arms on a radial maze. The rats were unable to discriminate the first arm they encountered from the other arms. Bird, W. A. Roberts, Abroms, Kit, and Crupi (2003) induced rats to carry food items (cheese and pretzels) from the center of a radial maze to boxes at the end of each arm; the rats were removed from the maze arm before the food was fully consumed. The rats hid and retrieved cheese preferentially over pretzels. However, the rats were insensitive to pilfering of the food. When Bird et al. degraded cheese at one delay interval but not the other, the rats did not selectively avoid locations with the degraded food. Taken together, these experiments by W. A. Roberts and colleagues provide evidence that the rats have knowledge of what type of food they hid and where they hid it, but no evidence that they remember when they hid it.

In contrast, Eichenbaum and colleagues (e.g., Fortin, Agster, \& Eichenbaum, 2002) have documented that rats quickly learn the sequence of randomly ordered odors. After being presented with a sequential order, the rats learned to select an odor that had appeared earlier in the sequence. The rats were then divided into hippocampal lesion or sham groups. The rats with the hippocampal lesions performed as well as the control rats on an odor recognition task, but they were impaired on tests of sequential 
order. These data are consistent with the hypothesis that the hippocampus is involved in memory for sequences of events. Eichenbaum and Fortin (2003) argued that memory for the sequential order of unique events provides a model for episodic memory.

Episodic memory is the latest kind of memory to develop and the first to degenerate with age (Tulving, 2002). Patients with neurodegenerative diseases such as Alzheimer's disease show a loss of episodic memory. Development of a rodent model for episodic memory could lead to a better understanding of the neural, molecular, and behavioral mechanisms of episodic memory (Griffiths \& Clayton, 2001). The availability of a rodent model could also permit the screening of putative pharmacotherapies for human memory disorders such as Alzheimer's (Clayton \& Griffiths, 2002) and would be a possible step toward the treatment and prevention of diseases involving the loss of episodic memory.

\section{REFERENCES}

BABB, S. J., \& Crystal, J. D. (2005). Discrimination of what, when, and where: Implications for episodic-like memory in rats. Learning \& Motivation, 36, 177-189.

Bird, L. R., Roberts, W. A., Abroms, B., Kit, K. A., \& Crupi, C. (2003). Spatial memory for food hidden by rats (Rattus norvegicus) on the radial maze: Studies of memory for where, what, and when. Journal of Comparative Psychology, 117, 176-187.

Church, R. M., Meck, W. H., \& GibBon, J. (1994). Application of scalar timing theory to individual trials. Journal of Experimental Psychology: Animal Behavior Processes, 20, 135-155.

Clayton, N. S., Bussey, T. J., \& Dickinson, A. (2003). Can animals recall the past and plan for the future? Nature Reviews Neuroscience, 4, 685-691.

Clayton, N. S., Bussey, T. J., Emery, N. J., \& Dickinson, A. (2003). Prometheus to Proust: The case for behavioural criteria for "mental time travel." Trends in Cognitive Sciences, 7, 436-437.

Clayton, N. S., \& Dickinson, A. (1998). Episodic-like memory during cache recovery by scrub jays. Nature, 395, 272-274.

Clayton, N. S., \& Dickinson, A. (1999a). Memory for the content of caches by scrub jays (Aphelocoma coerulescens). Journal of Experimental Psychology: Animal Behavior Processes, 25, 82-91.

Clayton, N. S., \& Dickinson, A. (1999b). Scrub jays (Aphelocoma coerulescens) remember the relative time of caching as well as the location and content of their caches. Journal of Comparative Psychology, 113, 403-416.

Clayton, N. S., \& Griffiths, D. P. (2002). Testing episodic-like memory in animals. In L. R. Squire \& D. L. Schacter (Eds.), Neuropsychology of memory (3rd ed., pp. 492-507). New York: Guilford.

Clayton, N. S., Yu, K. S., \& Dickinson, A. (2001). Scrub-jays (Aphelocoma coerulescens) form integrated memories of the multiple features of caching episodes. Journal of Experimental Psychology: Animal Behavior Processes, 27, 17-29.

Clayton, N. S., Yu, K. S., \& Dickinson, A. (2003). Interacting cache memories: Evidence for flexible memory use by western scrub-jays
(Aphelocoma californica). Journal of Experimental Psychology: Animal Behavior Processes, 29, 14-22.

CRYstAL, J. D. (2002). Timing inter-reward intervals. Learning \& Motivation, 33, 311-326.

Crystal, J. D., \& BABB, S. J. (2006). Spatial memory in rats after 25 hours. Manuscript in preparation.

DE Kort, S. R., Dickinson, A., \& Clayton, N. S. (2005). Retrospective cognition by food-caching western scrub-jays. Learning \& Motivation, 36, 159-176.

Eichenbaum, H., \& Fortin, N. J. (2003). Episodic memory and the hippocampus: It's about time. Current Directions in Psychological Science, 12, 53-57.

Fortin, N. J., Agster, K. L., \& Eichenbaum, H. (2002). Critical role of the hippocampus in memory for sequences of events. Nature Neuroscience, 5, 458-462.

Griffiths, D. P., \& Clayton, N. S. (2001). Testing episodic memory in animals: A new approach. Physiology \& Behavior, 73, 1-8.

Griffiths, D. P., Dickinson, A., \& Clayton, N. S. (1999). Declarative and episodic memory: What can animals remember about their past? Trends in Cognitive Sciences, 3, 74-80.

Hampton, R. R., Hampstead, B. M., \& Murray, E. M. (2005). Rhesus monkeys (Macaca mulatta) demonstrate robust memory for what and where, but not when, in an open-field test of memory. Learning \& Motivation, 36, 245-259.

Mistlberger, R. E. (1994). Circadian food-anticipatory activity: Formal models and physiological mechanisms. Neuroscience \& Biobehavioral Reviews, 18, 171-195.

Roberts, W. A. (2002). Are animals stuck in time? Psychological Bulletin, 128, 473-489.

Roberts, W. A., \& Roberts, S. (2002). Two tests of the stuck-in-time hypothesis. Journal of General Psychology, 129, 415-429.

Shettleworth, S. J. (1998). Cognition, evolution, and behavior. New York: Oxford University Press.

Suddendorf, T., \& Busby, J. (2003). Mental time travel in animals? Trends in Cognitive Sciences, 7, 391-396.

Suddendorf, T., \& Corballis, M. C. (1997). Mental time travel and the evolution of the human mind. Genetic, Social, \& General Psychology Monographs, 123, 133-167.

Tulving, E. (1972). Episodic and semantic memory. In E. Tulving \& W. Donaldson (Eds.), Organization of memory (pp. 381-403). New York: Academic Press.

Tulving, E. (1983). Elements of episodic memory. New York: Oxford University Press.

Tulving, E. (2002). Episodic memory: From mind to brain. Annual Review of Psychology, 53, 1-25.

Tulving, E. (2005). Episodic memory and autonoesis: Uniquely human? In H. S. Terrace \& J. Metcalfe (Eds.), The missing link in cognition: Origins of self-reflective consciousness (pp. 3-56). New York: Oxford University Press

Tulving, E., \& Markowitsch, H. J. (1998). Episodic and declarative memory: Role of the hippocampus. Hippocampus, 8, 198-204.

Zentall, T. R. (2005). Animals may not be stuck in time. Learning \& Motivation, 36, 208-225.

Zentall, T. R., Clement, T. S., Bhatt, R. S., \& Allen, J. (2001). Episodic-like memory in pigeons. Psychonomic Bulletin \& Review, 8, 685-690.

(Manuscript received September 16, 2005; revision accepted for publication October 31, 2005.) 